\title{
Educação interprofissional e prática colaborativa na formação em educação física: reflexões de uma experiência na perspectiva da tutoria'
} Interprofessional education and collaborative practice in physical education training: reflections of an experience from the perspective of tutoring

\author{
Ameliane da Conceição Reubens-Leonidio ${ }^{a, b}$ \\ (D) https://orcid.org/0000-0002-4343-0161 \\ E-mail: ameliane.doutoradoळgmail.com \\ Talita Grazielle Pires de Carvalho ${ }^{b}$ \\ (D) https://orcid.org/0000-0003-1090-7303 \\ E-mail: talitagraziellepiresळgmail.com \\ Maria Bernadete de Cerqueira Antunes ${ }^{c}$ \\ (iD) https://orcid.org/0000-0002-6391-397X \\ E-mail: mbcantunesळgmail.com \\ Mauro Virgílio Gomes de Barros ${ }^{b, d}$ \\ (iD) https://orcid.org/0000-0003-3165-0965 \\ E-mail: mauro.barrosळupe.br
}

anstituto Federal de Pernambuco. Cabo de Santo Agostinho, PE, Brasil.

b Universidade de Pernambuco/ Universidade Federal da Paraíba. Programa Associado de Pós-Graduação em Educação Física. Recife. PE. Brasil.

' Universidade de Pernambuco. Faculdade de Ciências Médicas da Universidade de Pernambuco. Módulo Interprofissional em Saúde. Recife, PE, Brasil.

d Universidade de Pernambuco. Escola Superior de Educação Física. Recife, PE, Brasil.

\section{Correspondência}

Talita Grazielle Pires de Carvalho

R. Arnóbio Marquês, 310. Recife, PE, Brasil. CEP 50100-130.

\section{Resumo}

Esse artigo objetiva descrever as características do componente curricular "módulo interprofissional em saúde" oferecido aos estudantes de graduação da área de saúde na Universidade de Pernambuco e apresentar um relato das experiências vivenciadas em atividades de tutoria, com destaque para a análise do cenário do núcleo da educação física. Essa estratégia de educação interprofissional e de prática colaborativa funciona em dois momentos pedagógicos, concentração e dispersão, que possibilitam o estudante entender o processo colaborativo de trabalho na perspectiva da integralidade e do cuidado na atenção à saúde em rede. Durante a concentração, estudantes do primeiro período reconhecem as possibilidades de atuação de seu núcleo em articulação com os demais e têm contato com conceitos da saúde coletiva. Durante a dispersão, os estudantes constroem e executam ações interprofissionais em uma instituição de ensino vinculada à estratégia de saúde da família, sendo as práticas corporais estratégias corriqueiras. Percebe-se que há uma mudança de atitude sobre a possibilidade de atuação no Sistema Único de Saúde (SUS); entretanto, é preciso que os conteúdos da saúde coletiva, inclusive a interprofissionalidade e as práticas colaborativas, perpassem toda a trajetória de formação em educação física, propiciando a qualificação para a integralidade da atenção no SUS.

Palavras-chave: Formação Interprofissional; Educação Física; Sistema Único de Saúde.

10 presente trabalho foi realizado com apoio da Coordenação de Aperfeiçoamento de Pessoal de Nível Superior - Brasil (CAPES). 
This article aims to describe the characteristics of the curricular component "interprofessional health module", offered to undergraduate health students at the University of Pernambuco (UPE) and to present a report of experiences in tutoring activities, with particular emphasis on the analysis of the Physical Education core scenario. This strategy of interprofessional education and collaborative practice, pioneered in the state, works in two pedagogical moments (concentration and dispersion), providing the student with an understanding of the collaborative work process from the perspective of comprehensiveness and care in network health care. Students from the first period of courses in the health area recognize during the concentration the possibilities of their core activities in articulation with others and have contact with concepts of collective health. During the dispersion, students build and perform interprofessional actions, in an educational institution linked to the family health strategy, and body practices are commonplace strategies. It is noticed that there is a change in attitude about the possibility of acting in the Brazilian Unified Health System (SUS); however, the contents of collective health, including interprofessionality and collaborative practices, must permeate the entire training path in Physical Education, providing the qualification for comprehensiveness. of Attention in SUS.

Keywords: Interprofessional Training; Physical Education; Brazilian Unified Health System.
Os desafios no campo da saúde diante da complexidade das necessidades individuais e coletivas fizeram com que, ao longo dos últimos 30 anos desde a criação do Sistema Único de Saúde (SUS), fossem instituídas várias políticas e estratégias que visassem a promoção, proteção e recuperação da saúde. Podemos citar dentre elas a Política Nacional de Atenção Básica (PNAB) (Brasil, 2006a, 2012, 2017b) e a Política Nacional de Promoção da Saúde (PNPS) (Brasil, 2006b, 2014) enquanto referências importantes para promover ações nesse sentido no território brasileiro.

Mesmo passando por redefinições ao longo dos anos, ambas dão suporte à Estratégia de Saúde da Família (ESF), ao Núcleo Ampliado de Saúde da Família e Atenção Básica (NASF-AB) e ao Programa Saúde na Escola (PSE) (Brasil, 2007, 2017a). O PSE é uma estratégia de articulação entre o Ministério da Saúde e o Ministério da Educação na intenção de promover a integração e a articulação política para melhorar a qualidade de vida do escolar. A ESF, o NASF-AB e a PSE, por sua vez, são políticas de extrema importância na experiência apresentada neste relato.

Para a operacionalização das estratégias de ação na atenção à saúde, é necessário articular saberes e práticas através do trabalho em equipe que funcione de maneira interdisciplinar e colaborativa. Pretende-se superar a fragmentação do conhecimento e das hierarquias entre saberes e profissões, tendo a integralidade como principal norteador das ações (Feriotti, 20o9; Reeves; Xyrichis; Zwarenstein, 2018). Neste sentido, é importante ampliar as discussões no tocante à formação profissional e humana para saúde que, sobretudo, contemple a articulação ensino-serviçocomunidade de forma que possa haver diálogo entre as Instituições de Ensino Superior (IES) e o SUS.

Nas Diretrizes Curriculares Nacionais dos Cursos de Graduação da Área da Saúde, definidas na Resolução $n^{0} 569$ de 8 de dezembro de 2017 (Brasil, 2018b), evidencia-se, a partir dos seguintes aspectos, a intenção de que as IES formem trabalhadores para o SUS: (1) Defesa da vida e defesa do SUS; (2) Atendimento às necessidades sociais em saúde; (3) Integração 
ensino-serviço-gestão-comunidade; (4) Integralidade e Redes de Atenção à Saúde; (5) Trabalho interprofissional; (6) Projetos Pedagógicos de Cursos (PPC) e componentes curriculares coerentes com as necessidades sociais em saúde; (7) Utilização de metodologias de ensino que promovam a aprendizagem colaborativa e significativa; (8) Valorização da docência na graduação, do profissional da rede de serviços e do protagonismo estudantil; (9) Educação e comunicação em saúde; (10) Avaliação com caráter processual e formativo; (11) Pesquisas e tecnologias diversificadas em saúde; (12) Formação presencial e carga horária mínima para cursos de graduação da área da saúde. Tais aspectos reafirmam a prerrogativa institucional do SUS de garantir a integralidade da atenção, através da formação interprofissional.

Recortando as diretrizes tendo em mente a educação física enquanto núcleo profissional da saúde, na Resolução nº 6, de 18 de dezembro de 2018 (Brasil, 2018d), que institui as Diretrizes Curriculares Nacionais dos Cursos de Graduação em Educação Física, é possível identificar, no eixo comum que perpassa a proposta atual de formação, conteúdo para atender às necessidades sociais de saúde. No entanto, é apenas na formação de bacharéis que se delimita a possibilidade de intervenção do profissional de educação física no SUS, com a integração ensino-serviço-comunidade um dos pontos apresentados para atingir esse objetivo.

Percebe-se que há algumas aproximações entre as Diretrizes Curriculares Nacionais dos Cursos de Graduação da Área da Saúde e as Diretrizes Curriculares Nacionais dos Cursos de Graduação em Educação Física, mais especificamente as do bacharelado. No entanto, o documento da área de educação física ainda é superficial quando trata do trabalho interprofissional e das práticas colaborativas, citando apenas a possibilidade do profissional de educação física compor equipes multiprofissionais em variados espaços, incluindo a saúde.

O Programa Nacional de Reorientação da Formação Profissional em Saúde (Pró-Saúde) (Brasil, 2005) em conjunto com o Programa de Educação pelo Trabalho Para Saúde, em suas edições PET-Saúde (Brasil, 20o8), PET-Saúde/ GraduaSUS (Brasil, 2015) e, mais recentemente,
PET-Saúde/Interprofissionalidade (Brasil, 2018c) no nível da graduação e os Programas de Residências Multiprofissionais em Saúde na pós-graduação são iniciativas do Ministério da Saúde que visam aproximar a educação física do SUS. No entanto, nota-se ainda uma limitação de experiências interprofissionais e de práticas colaborativas na graduação, pois os programas, por vezes, tem participação restrita por não fazerem parte do currículo dos cursos. Neste sentido, algumas universidades já caminham para superar tal fragilidade. Os trabalhos de Batista (2012), Candido, Rossit e Oliveira (2018), Capozzolo et al. (2018), Ferreira et al. (2013), apresentam propostas nas quais a interprofissionalidade é um dos elementos estruturantes da formação em educação física.

Neste artigo, apresenta-se o relato de uma experiência vivenciada no âmbito da educação interprofissional e da prática colaborativa na formação em educação física. No relato, discutem-se as vivências experimentadas durante o período de estágio de docência do curso de doutorado em Educação Física realizado no Módulo Interprofissional em Saúde "Atenção à Saúde na Perspectiva do Cuidado, Integralidade da Atenção e Trabalho em Equipe”. O módulo INTER, como é popularmente conhecido, é uma estratégia de formação para saúde que envolve todos os estudantes no primeiro período de seis cursos de saúde da Instituição de Ensino Superior (IES). Nesse contexto, têm-se como objetivo descrever as características do componente curricular "módulo interprofissional em saúde" oferecido aos estudantes de graduação da área de saúde na Universidade de Pernambuco (UPE) e apresentar um relato das experiências vividas nas atividades de tutoria, com particular destaque para análise do cenário do núcleo da educação física.

\section{Reflexões sobre as profissões e sobre o trabalho em equipe no SUS: núcleo, campo e educação interprofissional}

Os conceitos de "núcleo" e de "campo" de saber vêm sendo utilizados há mais de duas décadas nos estudos que tratam o processo de trabalho no SUS. Para Campos (200o), o "núcleo" demarca a identidade 
de uma área ou prática profissional, enquanto o "campo" corresponde ao espaço entre as atribuições dos núcleos como um lugar comum a todos. É neste espaço que se apresenta a complexidade da atenção à saúde, no qual há o reconhecimento dos limites de cada núcleo. Portanto, é necessário um alinhamento entre as ações do núcleo e do campo.

Quando o trabalho em saúde ocorre de modo isolado, ou seja, quando se concentra apenas na nas tecnologias relativas ao núcleo de saber, a tendência é que surja uma competição entre núcleos profissionais que pode reverberar numa atenção à saúde desarticulada e fragmentada (Costa et al., 2018). Além disso, é evidente que um único núcleo profissional não consegue dar conta das complexidades que se apresentam no SUS, o que enfatiza a importância do trabalho em equipe.

No entanto, quando se fala de trabalho em equipe, pode-se remeter à noção do compartilhamento de um campo por vários núcleos, com a partilha apenas do mesmo espaço físico e do atendimento dos mesmos usuários (Reeves; Xyrichis; Zwarenstein, 2018). Essa ideia deu origem, por exemplo, à denominação "trabalho multiprofissional”. Para Peduzzi (2009), essa é uma dificuldade do trabalho em equipe, visto que este necessita de uma maior colaboração entre os núcleos profissionais. Assim, a autora destaca que o trabalho em equipe é formado por três vertentes: (1) integração; (2) mudanças da abordagem saúdedoença de forma a compreender a multicausalidade; e (3) alterações nos processos de trabalho. De forma interprofissional, o trabalho em equipe pode ser uma estratégia eficaz para uma maior resolubilidade das demandas e para a manutenção do compromisso com a integralidade da atenção à saúde.

Peduzzi e Agreli (2018) consideram que a educação interprofissional (EIP) pode contribuir com o acesso e com a qualidade da atenção na saúde. No entanto, esse ainda é um tema pouco trabalhado nas IES, sobretudo do ponto de vista da operacionalização na formação. Neste sentido, em 2010 a Organização Mundial de Saúde (OMS) lançou o "Marco para a Ação em Educação Interprofissional e Prática Colaborativa" de forma a incentivar a proposta de trabalho interprofissional, que ganha cada vez mais notoriedade nos espaços políticos e acadêmicos.

A EIP propõe uma aprendizagem conjunta, na qual os estudantes de diferentes cursos possam aprender sobre o "núcleo" do outro, identificando aproximações e distanciamentos com o seu próprio "núcleo". Dessa forma, ela não desconsidera a formação uniprofissional ou multiprofissional, mas se apresenta como uma proposta de construção de competências para o trabalho em equipe, com a importante função de provocar mudanças no processo de trabalho em saúde (Costa et. al., 2018). De acordo com Batista (2012), revisões sistemáticas da literatura têm mostrado os efeitos positivos da EIP na atenção à saúde.

Nesse contexto, os sistemas de saúde e de educação devem trabalhar de forma articulada a fim de coordenar as estratégias para a força de trabalho na saúde. Se o planejamento da força de trabalho na saúde e a elaboração de políticas estão integrados, a EIP e a prática colaborativa podem ser plenamente sustentadas (OMS, 2010).

\section{Educação interprofissional e prática colaborativa: "aprender juntos para trabalhar juntos"}

O conceito de EIP em Saúde que aqui utilizamos refere-se a uma perspectiva de formação capaz de repercutir em práticas colaborativas nas equipes de trabalho. A OMS (2010) utiliza o slogan do Centre for Advancement in Interprofessional Education (CAIPE) “ "Aprender juntos para trabalhar juntos" para apontar a necessidade de articulação entre os sistemas de saúde e de educação de forma a manter a excelência das ações. Assim, a potência da força de trabalho em saúde colaborativa e preparada para a prática se dá através do encontro entre a EIP e a prática colaborativa.

A EIP se materializa no processo formativo através de modelos variados, a depender da realidade dos cursos. De acordo com Costa et al. (2018), podese adotar o modelo teórico, com estímulo à leitura 
de textos sobre o trabalho em equipe e prática colaborativa, e/ou o modelo prático, com visitas, discussões sobre a realidade observada e intervenções de campo que favoreçam o desenvolvimento de competências colaborativas através da identificação de possibilidades manifestadas a partir das várias interpretações sobre o fenômeno. A EIP pode ser incorporada tanto de modo tardio, no final do processo formativo, em que já há o domínio de referenciais teóricos e técnicos do núcleo profissional, quanto de modo precoce, quando há a possibilidade de ser abordada logo no início da formação, considerando que quanto mais cedo os estudantes participam de atividades interprofissionais, mais efetiva pode ser a desconstrução de hierarquias entre as profissões.

Os autores destacam ainda que as iniciativas de EIP, enquanto componentes curriculares, podem ter caráter eletivo - o que, embora facilite as discussões por conta da possibilidade de escolha, não afeta todos os estudantes - bem como caráter obrigatório, alcançando todos os estudantes e podendo integrar o PPC. A EIP pode também ocorrer de modo parcial, acontecendo apenas em alguns momentos da formação, como uma aula ou evento, ou de modo total, através da constituição de disciplinas ou de módulos.

Desta forma, é possível identificar que algumas formatações permitem uma maior aproximação dos estudantes com os outros núcleos profissionais e com a realidade da atenção à saúde, o que facilita a incorporação da colaboração e/ou das práticas colaborativas ainda no processo formativo. Para que isso ocorra de modo eficaz, é necessário que haja a qualificação dos docentes e dos profissionais de saúde que já se encontram no campo.

Neste sentido, em 2017 a Organização PanAmericana da Saúde (OPAS) construiu um plano de ação para EIP no Brasil, que inclui, dentre as linhas de ação: o fortalecimento da EIP no contexto das graduações em saúde; a incorporação da discussão nas pós-graduações e residências em saúde; a adoção da EIP em saúde nos processos formativos da educação permanente em saúde, e mais especificamente no Programa Mais Médicos; o desenvolvimento docente para a EIP; o mapeamento das iniciativas de EIP no Brasil; o compartilhamento de experiências de EIP no trabalho em saúde; o apoio às publicações das experiências nacionais de EIP; e o apoio à eventos sobre educação e trabalho interprofissional em saúde (Brasil, 2018a). Portanto, é importante transformar a EIP em proposta curricular nas IES, pois, desse modo, o desenvolvimento de competências necessárias para a prática colaborativa poderá se desenvolver (Batista; Batista, 2016).

Reeves et al. (2013) consideram que a colaboração é um elemento essencial para a melhoria dos resultados na atenção à saúde, enquanto Peduzzi e Agreli (2018) expõem que a colaboração e a prática colaborativa pertencem a uma abordagem contingencial da noção de trabalho interprofissional, que visa superar as tradicionais formas do trabalho em equipe. Destaca-se, contudo, que a colaboração deve ser um elemento presente tanto na formação para o campo no trabalho em equipe, como para o núcleo, ao se considerar as articulações necessárias dentro de um mesmo núcleo profissional.

Costa et al. (2018) fazem uma diferenciação entre colaboração e prática colaborativa de modo que a colaboração é entendida como uma forma de trabalho em saúde na qual não há uma identidade compartilhada de equipe, com menor interdependência e integração entre profissionais, enquanto a prática colaborativa está centrada no usuário e em sua singularidade, sendo a materialização da colaboração no processo de trabalho em saúde cujo objetivo é a integralidade.

De acordo com a OMS (2010), a implementação e a execução da prática colaborativa entre equipes de saúde ocorrem por meio de mecanismos de apoio institucional, de definições de modelos de governança, da estruturação de protocolos e do compartilhamento de procedimentos operacionais. Além disso, é necessário o desenvolvimento de políticas que estimulem as práticas colaborativas, assim como a existência de mecanismos de cultura de trabalho que permitam a equipe definir metas e projetos terapêuticos, com diálogo eficiente e balanceamento de ações individuais e coletivas e mecanismos ambientais que organizem espaços de modo a facilitar o diálogo entre os membros da equipe e reduzir hierarquias.

Há de se reconhecer que a aproximação com esses mecanismos ainda no processo formativo facilita a transição da identidade de estudante 
para a identidade profissional que mantenha uma relação interprofissional e colaborativa no processo de trabalho. Na sequência do texto, pode-se acompanhar a materialização da experiência de EIP e prática colaborativa no Módulo INTER da UPE, com especial destaque para a formação do bacharelado em educação física.

\section{Módulo interprofissional em saúde na Universidade de Pernambuco $e$} seus mecanismos

O Módulo Interprofissional na Área de Saúde: "Atenção à Saúde na Perspectiva do Cuidado, Integralidade da Atenção e Trabalho em Equipe" da UPE, ou Módulo INTER, é uma proposta de EIP total cujo objetivo é propiciar ao estudante o entendimento sobre o processo de trabalho em equipe colaborativo na perspectiva da integralidade e do cuidado na atenção à saúde em rede. 0 módulo foi implementado no segundo semestre de 2013 como um modo precoce de EIP, pois é ofertado aos estudantes do primeiro período dos cursos de saúde da IES.

Trata-se de uma estratégia de formação de caráter eletivo para os bacharelados em ciências biológicas e educação física e de caráter obrigatório para os cursos de enfermagem, medicina, odontologia e saúde coletiva. O Módulo INTER utiliza a sincronia entre o modelo teórico e o modelo prático da EIP, proporcionando, além da aquisição de referenciais sobre a saúde coletiva e de conhecimento sobre a profissão escolhida, o primeiro contato com o SUS, permitindo a execução de ações de promoção da saúde através da prática colaborativa, em conformidade com a ESF, o NASF-AB e a PSE.

Para uma melhor compreensão do modus operandi dessa estratégia formativa, utilizaremos dois temas descritos pela OMS (2010) como mecanismos para o desenvolvimento e oferecimento da EIP: (1) os mecanismos curriculares, que incluem a logística, o cronograma, o conteúdo programático, a presença obrigatória, os objetivos compartilhados, os princípios de aprendizado para adultos, o aprendizado contextual e a avaliação; e (2) os mecanismos do educador, que envolvem a capacitação dos profissionais acadêmicos e de líderes, o apoio institucional, o compromisso gerencial e os resultados de aprendizado, como mostra a Figura 1.

\section{Figura I - Modus operandi da estratégia formativa do Módulo Interprofissional em Saúde da Universidade de Pernambuco}

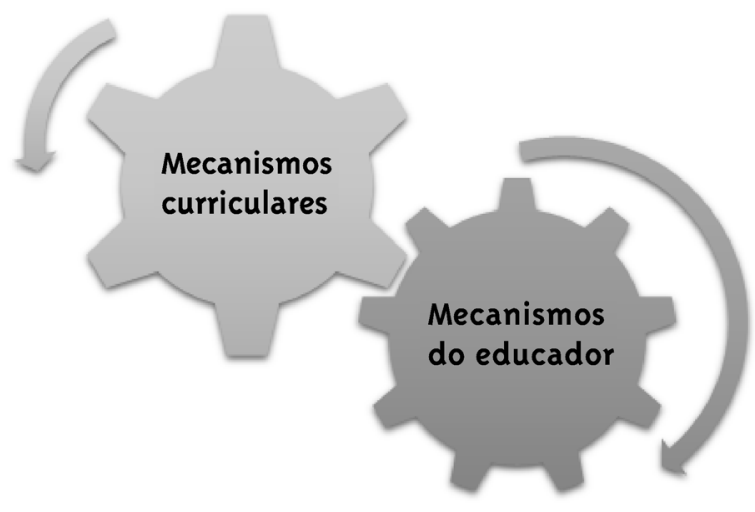

Nos mecanismos curriculares, em relação à logística, o Módulo INTER atende até 290 alunos por semestre distribuídos em 6 grupos organizados por cor (amarelo, azul, laranja, verde, rosa e roxo) com uma média de 49 alunos em cada. Os grupos constituídos de representações de todos os cursos são organizados em 6 subgrupos (A, B, C, D, E, F), acompanhados por três tutores. Desta forma, cada subgrupo pode trabalhar na perspectiva interprofissional e colaborativa em todas as atividades do semestre. Os estudantes têm acesso à operacionalização e ao conteúdo do Módulo INTER através de um site institucional. Por meio do site também é possível realizar a autoavaliação que trataremos mais adiante.

Os encontros são semanais e podem ocorrer em sala de aula, como atividade de concentração, ou no campo de intervenção, como atividade de dispersão. O cronograma é estruturado em 20 atividades teórico-práticas, seguindo o tempo do calendário acadêmico da UPE. O conteúdo programático é distribuído em dois momentos/unidades: o primeiro momento é o "Reconhecimento das profissões e do trabalho em equipe em saúde no SUS" e o segundo, a "Demonstração da capacidade de interagir e colaborar no trabalho em equipe na saúde". Durante a exposição do conteúdo programático são abordados o SUS e as profissões da saúde, os cursos 
de graduação da área da saúde, o conceito ampliado de saúde e a percepção de diferentes sujeitos sociais, e, por fim, o trabalho em equipe interprofissional e o processo de trabalho em saúde.

Os estudantes devem cumprir $75 \%$ da carga horária prevista e participar dos momentos de concentração e dispersão de forma ativa e colaborativa, a fim de desenvolver de modo integral os objetivos cognitivos, afetivos e psicomotores. Ao se considerar a problemática social, os princípios de aprendizagem são norteados pela abordagem crítica da realidade, apresentando-se como um caminho para aprendizagem contextual no próprio território de saúde do estado de Pernambuco, sobretudo no município de Recife.

Como referencial teórico estruturante do Módulo INTER, utiliza-se a obra "Pedagogia da Autonomia" de Freire (2004), levando-se em conta que, a partir da problematização, o estudante é capaz de estabelecer uma relação dialética entre ele e o objeto de aprendizagem. Assim, longe de utilizar manuais pré-determinados para o trabalho em equipe, nas atividades de concentração têm-se exposições dialogadas, leituras e debates, painéis com representação de cada núcleo profissional e interações. Embora o mesmo conteúdo seja ofertado em todos os grupos, as respostas são sempre diferentes e dinâmicas, revelando possibilidades amplas de olhares para o mesmo fenômeno.

Para as atividades de dispersão, em articulação com a ESF, NASF-AB e PSE, os estudantes, utilizando um roteiro de observação e de entrevistas, identificam um problema no espaço de intervenção, composto por escolas e/ou creches. Posteriormente, há uma teorização para construção e apresentação da proposta de intervenção para a comunidade escolar: diretoria, coordenação e docentes. No terceiro momento, há a execução do plano de intervenção com os alunos da educação básica. Desta forma, seguemse as etapas do Arco de Maguerez: observação da realidade, explicitação de pontos-chave, teorização, elaboração de uma hipótese de solução e aplicação na realidade (Prado et al., 2012).

Os estudantes passam por dois momentos avaliativos durante o semestre e que levam em conta três dimensões relacionadas com os objetivos da aprendizagem: a avaliação de conhecimentos, a avaliação de atitudes e a avaliação de habilidades. No entanto, eles não são avaliados unicamente pelos tutores, mas também por uma autoavaliação que contempla a dimensão das atitudes e a das habilidades que, por meio de diferentes pesos atribuídos a cada uma, resultam na média da unidade.

A avaliação dos conhecimentos realizada pelos tutores acontece tanto de forma coletiva como individual, através da redação de um diário de campo coletivo, do fichamento/resumo de textos, de arguições e seminários com pós-teste e da redação do trabalho final, que é um pôster. Destaca-se que a avaliação do pôster ocorre no formato científico, com dois pareceristas para cada trabalho que consideram os critérios de "escrita" e de “apresentação”. A avaliação de atitudes é individual e leva-se em consideração a comunicação interpessoal e em grupo, a organização do pensamento e da linguagem, a postura ética, o compromisso com a construção do conhecimento e a participação. A avaliação de habilidades também é individual e são observadas as competências adquiridas em relação à percepção do campo interprofissional e os papéis profissionais, à elaboração de estratégias e/ou materiais de contribuição da ação interprofissional na promoção da saúde e à demonstração da capacidade de interagir e colaborar no trabalho em equipe na saúde.

Por se tratar de uma experiência ainda no primeiro período do curso, o Módulo INTER, por meio de seus mecanismos curriculares, permite aos estudantes importantes aproximações com seu núcleo, com outros núcleos profissionais da saúde, com profissionais da atenção básica e com os profissionais da educação, possibilitando que eles apliquem a teoria ao mesmo tempo em que teorizam a prática em atividades de promoção à saúde junto a estudantes de escolas públicas.

Tratando-se dos mecanismos do educador, o Módulo INTER é composto pela coordenação geral, 18 tutores (professores da universidade, profissionais de saúde, residentes e discentes de pósgraduação stricto sensu), preceptores (profissionais de saúde que recebem os estudantes no território) e monitores (bolsistas dos cursos de graduação da UPE). Essa equipe abrange diferentes núcleos profissionais que dão suporte a todas as atividades 
desenvolvidas. O Módulo INTER é, inclusive, um espaço para formação docente de residentes e pósgraduandos stricto sensu. Para capacitar a equipe, ocorre um processo de educação permanente no início e no decorrer do semestre, através de encontros semanais nos quais são discutidos os temas que serão trabalhados com os estudantes, a aplicação das metodologias na prática pedagógica e a avaliação das ações de promoção da saúde, dentre outros pontos emergentes. Ao final do semestre, a equipe participa de um encontro de avaliação do processo formativo e dos resultados de aprendizado.

\section{Educação física no módulo interprofissional em saúde: percepções a partir do olhar de tutores}

O curso de bacharelado em Educação Física da UPE, que funciona na Escola Superior de Educação Física (ESEF) com duração de oito semestres distribuídos em quatro anos, com carga horária total de 3.258 horas, destina-se à oferecer uma:

formação acadêmica e profissional generalista, humanista e crítica qualificadora de uma intervenção fundamentada no rigor científico, na reflexão filosófica e na postura ética, na busca constante da relação teoria prática; nos conhecimentos biológicos, socioculturais, filosóficos, científicos, pedagógicos e técnicos; através de uma atuação, criativa e compromissada com uma sociedade plural e democrática. (UNIVERSIDADE DE PERNAMBUCO, 2017 p. 10)

Dentre as disciplinas eletivas disponibilizadas pelo curso está o Módulo INTER, oferecido para os alunos do primeiro período. Enquanto tutoras do módulo, além da participação nos momentos de educação permanente interprofissional e da avaliação do processo formativo junto com a coordenação e os demais tutores, foram realizadas sistematicamente discussões coletivas sobre o núcleo da educação física após cada encontro, atividade e/ ou avaliação, utilizando como instrumentos nossas observações em aula e as fichas de acompanhamento discente referentes à aquisição dos conhecimentos, atitudes e habilidades. Essas informações foram registradas e organizadas em formato de relatório, entregues ao final de cada semestre ao programa de pós-graduação ao qual estávamos vinculados. Dessa forma, o conteúdo desses materiais serviu de base para a construção do presente relato.

Foi observada uma certa desmotivação inicial dos estudantes de educação física, sobretudo pela pouca identificação deles com o SUS. Muitos chegam à formação inicial com um modelo pré-estabelecido de intervenção que atende às expectativas de mercado, como clubes e academias de ginástica, e não visualizavam as possibilidades de inserção nas políticas públicas, sobretudo as de saúde. Notou-se, contudo, que a maioria dos estudantes desconhecia as diretrizes do seu curso.

No cronograma do Módulo INTER, reserva-se um momento para o estudo das Diretrizes Curriculares Nacionais e do PPC do próprio curso, levando-os a ampliar as perspectivas com relação às possibilidades de atuação da educação física. 0 contato com tutores do seu próprio núcleo profissional também desperta nos estudantes uma certa curiosidade sobre as possibilidades de atuação. Neste sentido, o Módulo INTER torna-se um terreno fértil para descobertas da própria identidade profissional.

Por outro lado, os estudantes de educação física demonstravam certa expectativa favorável com relação ao trabalho em equipe com outros estudantes em formação para atuação na saúde e à possibilidade de desenvolver ações de promoção da saúde num cenário real. Nas intervenções, durante a dispersão no módulo interprofissional é comum observar a utilização de práticas corporais como um recurso de promoção e educação em saúde em escolas ou creches. Considerando-se a realidade escolar, fez-se necessária a adequação da estratégia utilizada e da faixa etária assim, práticas corporais são utilizadas em praticamente todas as temáticas abordadas. Contudo, é preciso lembrar que os estudantes ainda não possuem referenciais teóricos de seu núcleo definidos, sobretudo em relação à metodologias de ensino; logo, a utilização de jogos e brincadeiras adveio de conhecimentos prévios.

Os conhecimentos de núcleo são apreendidos na formação uniprofissional, mas é importante que se mantenha uma interface com a saúde coletiva no 
sentido de qualificar posteriores intervenções, seja enquanto estagiários, seja como trabalhadores do SUS. Não há instrumentos para uma análise mais detalhada deste trabalho, porém Costa et al. (2018) destaca que a experiência, sobretudo da prática colaborativa entre estudantes em formação de diferentes núcleos realizada de modo precoce no momento em que estão iniciando a jornada acadêmica, pode favorecer a quebra de hierarquias entre as profissões e o entendimento de que é preciso se apropriar das diferentes ferramentas do seu próprio núcleo assim como conhecer os demais núcleos para que haja uma maior resolutividades das ações de campo.

Para além das intervenções do Módulo INTER, é importante destacar que a inserção da educação física no SUS se fortaleceu a cada publicação da PNPS, ganhando maior notoriedade com o NASF-AB e, em especial, com o Programa Academia da Saúde, que instituiu as práticas corporais e as atividades físicas como um dos eixos estruturantes cujo objetivo é “contribuir para a promoção da saúde e produção do cuidado e de modos de vida saudáveis da população a partir da implantação de polos com infraestrutura e profissionais qualificados" (Brasil, 2013 p. 37).

Desta maneira, a formação de profissionais de educação física que saibam trabalhar em equipe de modo interprofissional e colaborativo é crucial para que a engrenagem do sistema de saúde funcione de fato no sentido da integralidade. De nada adiantará uma formação com excelência no núcleo profissional, se no processo de trabalho ocorrer isolamentos e fragmentações, pois não haverá potência necessária para atender às demandas que se apresentam na complexidade do SUS.

Vale destacar que as políticas públicas precisam favorecer a inserção desses profissionais, por isso é necessário bastante diálogo entre o sistema de saúde e o sistema de educação. De acordo com o trabalho de Candido, Rossit e Oliveira (2018), poucos profissionais de educação física formados numa perspectiva de integralidade para a atuação no SUS encontram-se inseridos na rede de saúde, perpetuando, assim, uma lógica de mercado na formação deste núcleo. Sobre os egressos do Módulo INTER da UPE, ainda não há estudos para apontar as repercussões da estratégia formativa. No entanto, no ano de 2020 um concurso público da saúde que contemplava profissionais de educação física ocorreu em Recife, o que pode ter sido uma porta de entrada para esses profissionais já formados numa perspectiva interprofissional e colaborativa.

\section{Considerações finais}

A EIP e a prática colaborativa são instrumentos para a promoção da integralidade do cuidado no SUS. Esse artigo apresentou a experiência do Módulo INTER da UPE, que é um dos recursos possíveis de formação interprofissional para a saúde que considera a integração entre os diferentes cursos da área da saúde da IES. A experiência não é pioneira no Brasil, mas é a primeira do estado de Pernambuco e permanece vigente desde o ano de 2013, tendo, consequentemente, profissionais que foram estudantes no Módulo INTER inseridos no sistema de saúde.

Especificamente sobre a formação no bacharelado em educação física, percebe-se um inicial desconhecimento dos estudantes com relação às áreas de atuação. 0 estranhamento da possibilidade de atuação no SUS sendo amenizado à medida que se iniciam as atividades de dispersão e se reconhecem as possibilidades de utilização de conteúdos relacionados ao seu núcleo profissional. Ter como referência na tutoria e preceptoria profissionais de educação física qualificados através da educação permanente também pode auxiliar na quebra de modelos de atuação que correspondam apenas ao mercado fitness quando se considera o eixo da promoção da saúde.

Para o desenvolvimento das competências do trabalho interdisciplinar e colaborativo, podemos considerar o Módulo INTER como um ponto de partida, um disparador de ações e como um espaço de articulação em rede e entre ensino-serviçocomunidade. No entanto, para a efetivação dessas competências no processo de trabalho em saúde, faz-se necessário - além da operacionalização das Diretrizes Curriculares Nacionais dos Cursos de Saúde e Diretrizes Curriculares Nacionais dos Cursos de Educação Física - a existência de políticas de incentivo e de incorporação nas IES e do respaldo durante toda a formação, sobretudo no eixo da saúde, 
pelo conteúdo da saúde coletiva. Assim, defendemos a transição de uma formação voltada principalmente ao mercado para uma formação que possa fornecer à sociedade profissionais qualificados para melhor atender à população segundo a perspectiva de integralidade da atenção no SUS.

\section{Referências}

BATISTA, N.A. Educação Interprofissional em Saúde: Concepções e Práticas. Caderno FNEPAS, Rio de Janeiro, v. 2, p. 25-28, jan. 2012.

BATISTA, N.A.; BATISTA, S.H.S.S. Educação interprofissional na formação em Saúde: tecendo redes de práticas e saberes. Interface: Comunicação, Saúde, Educação, Botucatu, v. 20, n. 56, p. 202-204, 2016. DOI: 10.1590/1807-57622015.0338

BRASIL. Ministério da Saúde. Pró-Saúde: Programa Nacional de Reorientação da Formação Profissional em Saúde. Brasília, DF, 2005.

BRASIL. Ministério da Saúde. Secretaria de Atenção à Saúde. Departamento de Atenção Básica. Política Nacional de Atenção Básica. Brasília, DF, 2006a.

BRASIL. Ministério da Saúde. Portaria nº 687, de 30 de março de 2006. Aprova a Política Nacional de Promoção da Saúde. Diário Oficial da União, Brasília, DF, 31 mar. 2006b. Seção 2, p. 138.

BRASIL. Decreto ${ }^{0}$ 6.286, de 5 de dezembro de 2007. Institui o Programa Saúde na Escola - PSE, e dá outras providências. Diário Oficial da União, Brasília, DF, 6 dez. 2007. Seção 1, p. 2.

BRASIL. Ministério da Saúde. Ministério da Educação. Portaria Interministerial $n^{0} 1.802$, de 26 de agosto de 2008. Institui o Programa de Educação pelo Trabalho para a Saúde - PETSaúde. Diário Oficial da União, Brasília, DF, 27 ago. 2008. Seção 1, p. 27.

BRASIL. Ministério da Saúde. Secretaria de Atenção à Saúde. Departamento de Atenção Básica. Política Nacional de Atenção Básica. Brasília, DF, 2012.

BRASIL. Ministério da Saúde. Portaria nº 2.681, de 7 de novembro de 2013. Redefine o Programa
Academia da Saúde no âmbito do Sistema Único de Saúde (SUS). Diário Oficial da União, Brasília, DF, 8 nov. 2013. Seção 1, p. 37.

BRASIL. Ministério da Saúde. Portaria nº 2.446, de 11 de novembro de 2014. Redefine a Política Nacional de Promoção da Saúde (PNPS). Diário Oficial da União, Brasília, DF, 13 nov. 2014. Seção 1, p. 68.

BRASIL. Ministério da Saúde. Secretaria de Gestão do Trabalho e da Educação na Saúde. Edital no 13, de 28 de setembro de 2015. Seleção para o Programa de Educação Pelo Trabalho Para a Saúde: PET-Saúde/ GraduaSUS - 2016/2017. Diário Oficial da União, Brasília, DF 29 set. 2015. Seção 3, p. 126.

BRASIL. Ministério da Saúde. Ministério da Educação. Portaria Interministerial n ${ }^{0} 1.055$, de 25 de abril de 2017. Redefine as regras e os critérios para adesão ao Programa Saúde na Escola - PSE por estados, Distrito Federal e municípios e dispõe sobre o respectivo incentivo financeiro para custeio de ações. Diário Oficial da União, Brasília, DF, 26 abr. 2017a. Seção 1, p. 36.

BRASIL. Ministério da Saúde. Portaria n 2.436, de 21 de setembro de 2017. Aprova a Política Nacional de Atenção Básica, estabelecendo a revisão de diretrizes para a organização da atenção básica, no âmbito do sistema único de saúde (SUS). Diário Oficial da União, Brasília, DF, 22 set. 2017 b. Seção 1, p. 68.

BRASIL. Ministério da Saúde. Construindo caminhos possíveis para a Educação Interprofissional em Saúde nas Instituições de Ensino Superior do Brasil. Brasília, DF, 2018a.

BRASIL. Ministério da Saúde. Conselho Nacional de Saúde. Resolução ${ }^{0}{ }^{569}$, de 8 de dezembro de 2017. Princípios Gerais para as Diretrizes Curriculares Nacionais dos Cursos de Graduação da Área da Saúde. Diário Oficial da União, Brasília, DF, 26 fev. 2018b. Seção 1, p. 85.

BRASIL. Ministério da Saúde. Edital nº 10, de 23 de julho de 2018 para o Programa de Educação pelo Trabalho para a Saúde PET-Saúde/ Interprofissionalidade - 2018/2019. Diário Oficial União, Brasília, DF, 24 jul. 2018c. Seção 3, p. 78. 
BRASIL. Ministério da Saúde. Conselho Nacional de Educação. Câmara de Educação Superior. Resolução ${ }^{0}$ 6, de 18 de dezembro de 2018. Institui Diretrizes Curriculares Nacionais dos Cursos de Graduação em Educação Física e dá outras providências. Diário Oficial da União, Brasília, DF, 19 dez. 2018d. Seção 1, p. 48.

CAMPOS, G.W.S. Saúde pública e saúde coletiva: campo e núcleo de saberes e práticas. Ciência \& Saúde Coletiva, Rio de Janeiro, v. 5, n. 2, p. 219-23o, 2000. DOI: 10.1590/S1413-81232000000200002

CANDIDO, L.O.; ROSSIT, R.A.S.; OLIVEIRA, R.C. Inserção profissional dos egressos de um curso de educação física com ênfase na formação em saúde. Trabalho, Educação e Saúde, Rio de Janeiro, v. 16, n. 1, p. 305-318, 2018. DOI: 10.1590/1981-7746-solooog6

CAPOZZOLO, A.A. et al. Formação interprofissional e produção do cuidado: análise de uma experiência. Interface: Comunicação, Saúde, Educação, Botucatu, v. 22, n. 2, p. 1675-1684, 2018. Suplemento. DOI: 10.1590/1807-57622017.0679 COSTA, M.V. et. al. Educação interprofissional em saúde. Natal: SEDIS-UFRN, 2018.

FERREIRA, S.E. et al. Formação profissional em Educação Física e saúde na Universidade Federal de São Paulo. Revista Brasileira de Atividade Física e Saúde, Pelotas, v. 5, n. 18, p. 646-651, 2013. DOI: $10.12820 /$ rbafs.v.18n5p646.

FERIOTTI, Maria de Lourdes. Equipe multiprofissional, transdisciplinaridade e saúde: desafios do nosso tempo. Vínculo, São Paulo, v. 6, n. 2, p. 179-190, 2009.

FREIRE, P. Pedagogia da autonomia: saberes necessários à prática educativa. São Paulo: Paz e Terra, 2004.
OMS - ORGANIZAÇÃO MUNDIAL DA SAÚDE. Marco para Ação em Educação Interprofissional e Prática Colaborativa. Genebra, 2010.

PEDUZZI, M. Trabalho em equipe. In: FUNDAÇÃO OSWALDO CRUZ; ESCOLA POLITÉCNICA DE SAÚDE JOAQUIM VENÂNCIO. Dicionário da Educação em Saúde. Rio de Janeiro, 2009. Disponível em: <http://www.sites.epsjv.fiocruz.br/dicionario/ verbetes/traequ.html $>$. Acesso em: 19 jul. 2020.

PEDUZZI, M; AGRELI, H.F. Trabalho em equipe e prática colaborativa na Atenção Primária à Saúde. Interface: Comunicação, Saúde, Educação, Botucatu, v. 22, n. 2, p. 1525-1534, 2018. Suplemento. DOI: 10.1590/1807-57622017.0827

PRADO, M.L. et al. Arco de Charles Maguerez: refletindo estratégias de metodologia ativa na formação de profissionais de saúde. Escola Anna Nery, Rio de Janeiro, v. 16, n. 1, p. 172-177, 2012. DOI: 10.1590/S1414-81452012000100023

REEVES, S. et al. Interprofessional education: effects on professional practice and healthcare outcomes (update). The Cochrane Database of Systematic Reviews, Oxford, v. 2013, n. 3, CDoo2213, 2013. DOI: 10.1002/14651858.

CDoo2213.pub3

REEVES, S.; XYRICHIS, A.; ZWARENSTEIN, M. Teamwork, collaboration, coordination, and networking: Why we need to distinguish between different types of interprofessional practice. Journal of Interprofessional Care, Abingdon, v 32, p. 1-3, 2018. DOI: 10.1080/13561820.2017.1400150

UNIVERSIDADE DE PERNAMBUCO. Escola Superior de Educação Física. Projeto Pedagógico de Curso: bacharelado em Educação Física: processo de renovação de reconhecimento de curso. Recife, 2017.

\section{Contribuição dos autores}

Reubens-Leonidio e Carvalho contribuiram com a concepção, redação do artigo, revisão crítica e aprovação da versão a ser publicada. Antunes e Barros realizaram a revisão crítica e aprovação da versão a ser publicada.

Recebido: $24 / 12 / 2020$

Reapresentado: $24 / 12 / 2020$

Aprovado: 29/03/2021 